\title{
QUADRATIC FORMS PERMITTING TRIPLE COMPOSITION BY
}

\author{
KEVIN MCCRIMMON
}

\begin{abstract}
In an algebraic investigation of isoparametric hypersurfaces, J. Dorfmeister and E. Neher encountered a nondegerate quadratic form which permitted composition with a trilinear product, $Q(\{x y z\})=Q(x) Q(y) Q(z)$. In this paper we give a complete description of such composition triples: they are all obtained as isotopes of permutations of standard triples $\{x y z\}=(x y) z$ or $x(y z)$ determined by a composition algebra, with the quadratic form $Q$ the usual norm form. For any fixed $Q$ this leads to 1 isotopy class in dimensions 1 and 2, 3 classes in the dimension 4 quaternion case, and 6 classes in the dimension 8 octonion case.
\end{abstract}

Throughout we work with finite-dimensional vector spaces $X$ over a field $\Phi$ of arbitrary characteristic. (Charactristic 2 will cause us a few headaches, and we sometimes pass to an infinite extension $\Omega \supset \Phi$ to perform linearizations, but otherwise the field plays no role in the arguments.) A quadratic form is nondegenerate if $\operatorname{Rad}(Q)=\{z \mid Q(z)=Q(z, X)=0\}$ vanishes, and is nonsingular if the symmetric bilinear form $Q(x, y)=Q(x+y)-Q(x)-Q(y)$ is nondegenerate, i.e. $\operatorname{Bilrad}(Q)=\{z \mid Q(z, X)=0\}$ vanishes. These two concepts coincide if the characteristic $\neq 2$. A triple system $T=(X,\{\})$ consists of a vector space $X$ together with a triple product, which is simply a trilinear map $X \times X \times X \rightarrow X$; it is not assumed to be in any sense commutative or associative or unital. A composition triple $(T, Q)$ is a triple system $T$ together with a nondegenerate form $Q$ which permits composition

$$
Q(\{x y z\})=Q(x) Q(y) Q(z) \text {. }
$$

Note that if $Q$ permits composition so does $-Q$, and that $\alpha Q$ permits composition with respect to the triple product $\alpha\{x y z\}$.

Two triple systems $T, T^{\prime}$ are isomorphic (written $T \cong T^{\prime}$ ) if there is a linear bijection $F: X \rightarrow X^{\prime}$ with

$$
F(\{x y z\})=\{F(x) F(y) F(z)\}^{\prime} .
$$

More generally, two systems are isotopic (written $T \sim T^{\prime}$ ) if there is a quadruple of linear bijections $F_{i}: X \rightarrow X^{\prime}(i=0,1,2,3)$ with

$$
F_{0}(\{x y z\})=\left\{F_{1}(x) F_{2}(y) F_{3}(z)\right\}^{\prime} .
$$

(For example, the scalar multiple $\{x y z\}^{\prime}=\alpha\{x y z\}$ is isotopic to $\{x y z\}$ via $F_{0}=$ $\alpha I, F_{1}=F_{2}=F_{3}=I$.) Given any quadruple of bijections and a triple product $\{x y z\}$ on $X$, we can induce a triple product on $X^{\prime}$ isotopic to the original via $\left\{x^{\prime} y^{\prime} z^{\prime}\right\}^{\prime}=$ $F_{0}\left\{F_{1}^{-1}\left(x^{\prime}\right) F_{2}^{-1}\left(y^{\prime}\right) F_{3}^{-1}\left(z^{\prime}\right)\right\}$. If $T$ is a composition triple and the $F_{i}$ are norm

Received by the editors September 30, 1981.

1980 Mathematics Subject Classification. Primary 17D05; Secondary 17A30, 17 A45. 
similarities $Q^{\prime}\left(F_{i}(x)\right)=\alpha_{i} Q(x)$ of $(X, Q)$ to $\left(X^{\prime}, Q^{\prime}\right)$ with $\alpha_{0}=\alpha_{1} \alpha_{2} \alpha_{3} \neq 0$, then the induced triple product on $X^{\prime}$ permits composition with $Q^{\prime}$. If $(T, Q),\left(T^{\prime}, Q^{\prime}\right)$ are composition triples then by an isotopy of composition triples we mean an isotopy of triple systems consisting of norm similarities with $\alpha_{0}=\alpha_{1} \alpha_{2} \alpha_{3} \neq 0$; an isomorphism of composition triples is the special case where all $F_{i}=F$ and all $\alpha_{i}=1$, i.e. an isometric isomorphism.

Thus we can obtain new composition triples from old ones via any quadruple of isometries. This suggests that we classify the composition triples up to isotopy rather than up to isomorphism.

In the classical theory of composition algebras due to Albert, Kaplansky, and Jacobson, a general algebra permitting composition is shown to be isotopic to a unital one, and the unital composition algebras are shown to be isomorphic to 4 types. Similarly, for composition triples the arbitrariness of isotopy is needed for nonunital systems: once a composition triple is unital in the sense that is has a unit element $u \in X$ with

$$
\{u u x\}=\{u x u\}=\{x u u\}=x \text { for all } x \in X
$$

then it can be described up to isomorphism. Note that if $T$ is a unital composition triple then (0.4) and (0.1) imply $Q(u)^{2}=1$, so $Q(u)= \pm 1$; replacing $Q$ by $-Q$ if necessary, we can always assume the unit has

$$
Q(u)=1 \text {. }
$$

The unit element $u$ is certainly not unique: $-u$ will do just as well.

We make frequent use of the linearization principle that any identity which holds identically (in the sense that it remains valid in all scalar extensions $T_{\Omega}=T \otimes_{\Phi} \Omega$, not just in $T$ itself) can be linearized: we can replace $x$ by $x+\lambda y$ and equate coefficients of $\lambda$ in the resulting expression (e.g. over $\Phi(\lambda)$ the powers of the indeterminate $\lambda$ are independent over $\Phi)$. Note that $(0.1)$ and all consequences thereof hold identically in a composition triple, since $(0.1)$ is quadratic in all its variables, and quadratic identities remain valid in all scalar extensions.

We will also on occasion make use of the density principle that to prove a certain polynomial relation $F\left(x_{1}, \ldots, x_{n}\right)=0$ on a composition triple, it suffices to prove it for a Zariski-dense set of $x_{i}$ 's. This of course is the whole point of the Zariski topology, but it makes sense only for infinite fields. Rather than pass to an infinite extension in the middle of the proof, let us observe once-and-for-all that to prove $F=0$ on $T$ it suffices to prove $F=0$ on an infinite extension $T_{\Omega}$, and in this extension it is enough to establish the relation on some dense set.

1. Composition algebras. We begin by reviewing the properties of composition algebras we will need in the sequel. A composition algebra $(C, Q)$ consists of an algebra $C=(X, \cdot)$ together with a nondegenerate quadratic form (called the norm form) which permits composition,

$$
Q(x \cdot y)=Q(x) Q(y) .
$$

Two composition algebras $C, C^{\prime}$ are isotopic if there exists a triple $F_{0}, F_{1}, F_{2}$ of norm similarities $(X, Q) \rightarrow\left(X^{\prime}, Q^{\prime}\right)$ such that

$$
F_{0}(x \cdot y)=F_{1}(x) \cdot{ }^{\prime} F_{2}(y), \quad Q^{\prime}\left(F_{i}(x)\right)=\alpha_{i} Q(x), \quad \alpha_{0}=\alpha_{1} \alpha_{2} \neq 0 .
$$


A composition algebra is unital if it has a unit element $u$ with $u \cdot x=x \cdot u=x$ for all $x$, in which case (1.1) forces $Q(u)=1$. Any composition algebra is isotopic to a unital one [3,p. 957]: if $Q(u) \neq 0$ then $u_{1} \cdot u_{2}=u$ implies the left and right multiplications $L\left(u_{1}\right), R\left(u_{2}\right)$ are bijective norm similarities, and $x \cdot{ }_{u} y=R\left(u_{2}\right)^{-1} x$. $L\left(u_{1}\right)^{-1} y$ has unit $u$ and permits composition with respect to $Q_{u}(x)=Q(u)^{-1} Q(x)$. (This passage to a unital isotope is the only place where finite-dimensionality is needed.) We make repeated use of the following basic facts about unital composition algebras $C$ (see $[1,3,4])$; denoting the unit by 1 , we have

$\bar{x}=T(x) 1-x$ for $T(x)=Q(x, 1)$ defines an involution on $C$ with the properties

(i) $Q(x y, z)=Q(y, \bar{x} z)=Q(x, z \bar{y})$,

(ii) $x \bar{x}=\bar{x} x=Q(x) 1$, so $x$ is invertible iff $Q(x) \neq 0$, in which case $x^{-1}=Q(x)^{-1} \bar{x}$,

(iii) $x y x=Q(x, \bar{y}) x-Q(x) \bar{y}$,

(iv) $x^{2}=T(x) x-Q(x) 1$.

(1.4) The composition algebras are built up by the Cayley-Dickson process: if $B$ is any proper subalgebra of $C$ on which the norm is nondegenerate, then $C \supset B \oplus B l$ with

(i) $\left(b_{1} \oplus c_{1} l\right) \cdot\left(b_{2} \oplus c_{2} l\right)=\left(b_{1} b_{2}+\mu \bar{c}_{2} c_{1}\right) \oplus\left(c_{2} b_{1}+c_{1} \bar{b}_{2}\right) l$,

(ii) $Q(b \oplus c l)=Q(b)-\mu Q(c) . \quad \overline{b \oplus c l}=\bar{b}-c l$

for any $l \in B^{\perp}$ with $Q(l)=-\mu \neq 0$.

(1.5) Every composition algebra is one of 4 types.

Type I. A purely inseparable field extension $\Sigma$ of $\Phi$ of exponent 1, with $Q(\sigma)=\sigma^{2}$ (so $\Sigma=\Phi$ has dimension 1 if $\Phi$ has characteristic $\neq 2$, but $\Sigma$ may have arbitrary dimension in characteristic 2). The involution on $\Sigma$ is trivial, $\bar{\sigma}=\sigma$.

Type II. A quadratic extension $\Omega$ of dimension 2, determined in characteristic $\neq 2$ by the Cayley-Dickson process from $\Phi 1(\Omega=\Phi 1 \oplus \Phi i$ for $T(i)=0, Q(i) \neq 0)$, while in characteristic $2 \Omega=\Phi 1 \oplus \Phi \omega$ with $T(\omega)=1 . \Omega$ is commutative and associative, but the involution is nontrivial.

Type III. A quaternion algebra $B=\Omega \oplus \Omega j$ of dimension 4 determined by the Cayley-Dickson process from a quadratic extension $\Omega . B$ is associative but not commutative.

Type IV. An octonion algebra $C=B \oplus B l$ of dimension 8 determined by the Cayley-Dickson process from a quaternion algebra $B . C$ is not associative.

(1.6) The norm form $Q$ of a composition algebra is nonsingular in all cases except Type $I$ in characteristic 2 , in which case the bilinear form $Q(x, y)$ vanishes identically.

In particular, all unital composition algebras are alternative algebras in the sense that the associator $[x, y, z]=(x y) z-x(y z)$ is an alternating function of its three 
arguments; such algebras also satisfy the left, right, and middle Moufang identities

$$
\begin{aligned}
& \text { (i) }(x y x) z=x(y(x z)), \\
& \text { (ii) } z(x y x)=((z x) y) x \quad(x y x=(x y) x=x(y x)) \text {, } \\
& \text { (iii) } x(y z) x=(x y)(z x)
\end{aligned}
$$

and the inverse identities

$$
x\left(x^{-1} y\right)=y=\left(y x^{-1}\right) x \quad \text { whenever } x x^{-1}=x^{-1} x=1
$$

as well as the bumping identities

$$
[x, y, x z]=[x, y, z] x, \quad[x, y, z x]=x[x, y, z] .
$$

"Unital isotopy" takes a very simple form for composition algebras $C$ :

(1.10) if $t$ is an invertible element in a composition algebra $C$ then the elemental $t$-isotope

(i) $C^{(t)}: x \cdot{ }_{t} y=\left(x t^{-1}\right)(t y)$

is again a composition algebra with the same unit and inversion map; elemental isotope is an equivalence relation,

(ii) $\left\{C^{(t)}\right\}^{(s)}=C^{(t s)}$,

(iii) $\left\{C^{(t)}\right\}^{\left(t^{-1}\right)}=C^{(1)}=C$.

(1.11) If $C$ and $C^{\prime}$ are composition algebras on $X$ with the same unit and norm, then $C^{\prime}$ is an elemental isotope or anti-isotope of $C$ :

$$
x \cdot{ }^{\prime} y=\left(x t^{-1}\right)(t y) \text { or } x \cdot{ }^{\prime} y=\left(y t^{-1}\right)(t x) .
$$

Since (1.11) is not so well known, we sketch a proof: the Cayley-Dickson process shows a composition algebra is determined by its unit and norm form, so $C^{\prime}$ is isomorphic to $C, F\left(x \cdot{ }^{\prime} y\right)=F(x) F(y)$ for some isometry $F$ (we do not have $C^{\prime}=C$, because even if $B^{\prime}=B$ and $l^{\prime}=l$ in the Cayley-Dickson process we need not have $\left.b \cdot{ }^{\prime} l=b \cdot l\right)$; but isometries satisfy the triality principle $G(x y)=$ $G_{1}(x) G_{2}(y)$ or $G_{2}(y) G_{1}(x)$ [5, Theorem 1, p.159], so for $G=F^{-1}$ we have $x \cdot{ }^{\prime} y=$ $F_{1}(x) F_{2}(y)$ or $F_{2}(y) F_{1}(x)$; in the former case $x=1, y=1$ show $F_{2}(y)=$ $t_{1}^{-1} y, F_{1}(x)=x t_{2}^{-1}$ for $t_{i}=F_{i}(1), t_{1} t_{2}=1$, so $x \cdot{ }^{\prime} y=\left(x t^{-1}\right)(t y)$ for $t=t_{2}=t_{1}^{-1}$, and similarly in the latter case.

2. Certain impossibilities. For our later work we need to rule out certain identities involving elements $t, s$ in an octonion algebra. Since the identities are of degree 5 in elements $x, y$ it will make the calculations more manageable to be able to take $t$ from a quaternion subalgebra, or to take $t=l$. We first show this entails no loss of generality.

2.1 Imbedding Lemma. Let $t$ be an element of an octonion algebra $C$.

(i) If $t \notin \Phi 1$ is invertible with $T(t)=0$, then it can be imbedded as $t=l$ in $C=B \oplus B l$;

(ii) $t$ can always be imbedded in a quaternion subalgebra, $C=B \oplus B l$ with $t \in B$; if $t \notin \Phi 1$ we can find a basis $1, t, v$, tv for $B$ with $Q(v) \neq 0, T(v)=0$. 
Proof. (i) $t^{\perp}$ has dimension 7 and contains 1 by hypothesis $T(t)=0$; choose $u \perp t$ so $\Omega=\Phi 1 \oplus \Phi u$ is nonsingular of type (1.5)II orthogonal to $t$ (i.e. $T(u)=0 \neq$ $Q(u)$ in characteristic $\neq 2, T(u)=1$ in characteristic 2$)$. Then $W=\{1, u, t, \bar{u} t\}^{\perp}=$ $\{\Omega+\Omega t\}^{\perp}$ has dimension $\geqslant 4$. If $W$ is totally isotropic then

$$
W \subset W^{\perp}=\{\Omega+\Omega t\}^{\perp \perp}=\Omega+\Omega t
$$

by nonsingularity of $Q$, where the former has dimension $\geqslant 4$ and the latter dimension $\leqslant 4$, hence $W=\Omega+\Omega t$ is 4-dimensional maximal isotropic, contrary to $\Omega$ being nonsingular. Thus $w \in W$ exists with $Q(w) \neq 0$, and $B=\Omega+\Omega w$ is a quaternion subalgebra of type (1.5)III orthogonal to $t$ (note $w \perp t, \bar{u} t \Rightarrow w, u w \perp t \Rightarrow$ $B \perp t$ by (1.3)(i)), hence by (1.4) $C=B \oplus B t$.

(ii) All is trivial if $t \in \Phi 1$, so assume $t \notin \Phi 1$. The subalgebra $\Omega=\Phi 1 \oplus \Phi t$ will have type (1.5)II if $Q$ is nondegenerate on $\Omega$, and in this case by (1.4) $\Omega$ is imbeddable in a quaternion algebra $B=\Omega \oplus \Omega j$ with basis $1, t, j, t j$ for $Q(j) \neq 0$ $=T(j)$. If $Q$ is degenerate on $\Omega$, so $Q(z)=T(z)=0$ for some $z \in \Omega$ (necessarily $z \notin \Phi 1$ ), choose $v \in C$ with $T(v)=Q(v, 1)=0, Q(v, z)=-1$ and $Q(v) \neq 0$ (if our initial $v$ has $Q(v)=0$ replace it by $v^{\prime}=v+w$ where $w \in W=\{1, z, v\}^{\perp}$ has $Q(w) \neq 0$, noting that $W$ of dimension $\geqslant 5$ cannot be totally isotropic). Then $B=\Phi 1+\Phi z+\Phi v+\Phi z v=\Omega+\Omega v$ is a subalgebra (1, x,y, xy always spans a subalgebra by (1.3)(iii), (1.3)(iv) and its linearization $x y+y x=T(x) y+$ $T(y) x-Q(x, y) 1)$, on which $Q$ is nonsingular: relative to the basis $1, z, v, z v$ the bilinear form has discriminant

$$
\operatorname{det}\left[\begin{array}{cccc}
2 & 0 & 0 & 1 \\
0 & 0 & -1 & 0 \\
0 & -1 & 2 Q(v) & 0 \\
1 & 0 & 0 & 0
\end{array}\right]=1
$$

since $T(z)=T(v)=0, T(z v)=Q(\bar{z}, v)=-Q(z, v)=1, Q(z)=Q(z v)=$ $0, Q(z, z v)=Q(z) T(v)=0, Q(v, z v)=Q(v) T(z)=0$. Thus $B$ is a quaternion subalgebra with basis $1, t, v, t v$ where $v$ has $T(v)=0 \neq Q(v)$.

We will also need to be able to cancel certain expressions in a quaternion subalgebra.

2.2 CANCELling Lemma. If $B$ is a quaternion algebra then $Q(b, \bar{b})$ does not vanish for all $b \in B$, and $Q([b, t])$ vanishes for all $b \in B$ only if $t \in \Phi 1$ (i.e. if $t \notin \Phi 1$ then $[b, t]$ is invertible for a dense set of $b$ 's).

Proof. $Q(b, \bar{b})=T(b) Q(b, 1)-Q(b, b)=T(b)^{2}-2 Q(b)$ does not vanish since in characteristic 2 we can find $T(b)=1$ and in characteristic $\neq 2$ we can find invertible skew elements $T(b)=0 \neq Q(b)$.

Vanishing of $Q([b, t])$ means

$$
\begin{aligned}
0 & =Q([b, t])=Q(b t)-Q(b t, t b)+Q(t b) \\
& =2 Q(b) Q(t)-Q(b t,(t b+b t)-b t) \\
& =4 Q(b) Q(t)-Q(b t, T(t) b+T(b) t-Q(b, t) 1) \quad(\text { linearizing }(1.3)(\text { iv })) \\
& =4 Q(b) Q(t)-T(t)^{2} Q(b)-T(b)^{2} Q(t)+Q(b, t) Q(b t, 1) ;
\end{aligned}
$$


so from (1.3)(i)

$$
\left\{4 Q(t)-T(t)^{2}\right\} Q(b)-T(b)^{2} Q(t)+Q(b, t) Q(b, \bar{t})=0 .
$$

If this quadratic relation holds for all $b$ we can linearize $b \rightarrow b+\lambda c$ to get $0=Q\left(\left\{4 Q(t)-T(t)^{2}\right\} b-2 T(b) Q(t) 1+Q(b, t) \bar{t}+Q(b, \bar{t}) t, c\right)$ for all $c$, so by nonsingularity $0=\left\{4 Q(t)-T(t)^{2}\right\} b-2 T(b) Q(t) 1+Q(b, t) \bar{t}+Q(b, \bar{t}) t$, and since there exists $b \notin \Phi 1+\Phi t+\Phi \bar{t}$ we must have $4 Q(t)-T(t)^{2}=0$. Going back to $(*)$, we get $-T(b)^{2} Q(t)+Q(b, t) Q(b, \bar{t})=0$; since $t \notin \Phi 1$ we can find $b$ with $T(b)=Q(b, 1) \neq 0, Q(b, t)=0$, which forces $Q(t)=0$; but then $Q(b, t) Q(\bar{b}, t)=$ $Q(b, t) Q(b, \bar{t})=0$ forces $Q(B, t)=0$, hence $t=0$ by nonsingularity, contrary to $t \notin \Phi 1$.

Now we can establish the first impossibility.

2.3 First ImPossible IDENTITY. No two invertible nonscalar elements $t, s \notin \Phi 1$ in an octonian algebra can satisfy

$$
Q([x t, s, y],[x, t, s y] s \cdot y)=0
$$

identically in $x$ and $y$.

Proof. We assume (2.4) holds identically for $t \notin \Phi 1$ and prove this forces $s \in \Phi 1$. By (2.1) we may assume $C=B \oplus B l$ where $t$ lies in the quaternion subalgebra $B$. We write $s=u+v l$ for some $u, v \in B$.

First we show $s \notin B l$. Suppose on the contrary that $u=0, s=v l$. Linearizing $y \rightarrow y+\lambda s^{-1}$ in (2.4) and using $\left[C, s, s^{-1}\right]=\left[C, C, s s^{-1}\right]=0$ yields as coefficient of $\lambda$

$$
Q([x t, s, y],[x, t, s y])=0 .
$$

If we take $x=y=b \in B$ we see

$$
\begin{aligned}
0 & =Q([b t, v l, b],[b, t,(v l) b])=Q([b, b t, v l],[b, t,(v \bar{b}) l]) \\
& =Q(v[b, b t] l, v \bar{b}[b, t] l) \quad(\text { from }[b, c, d l]=\{d[b, c]\} l \text { by }(1.4)(\mathrm{i})) \\
& =-\mu Q(v b[b, t], v \bar{b}[b, t])=-\mu Q(v) Q(b, \bar{b}) Q([b, t]) .
\end{aligned}
$$

But by (2.2) $Q([b, t]) \neq 0$ and $Q(b, \bar{b}) \neq 0$ for a dense set of $b$ 's, forcing $Q(s)=$ $Q(v l)=-\mu Q(v)=0$, contrary to the invertibility of $s$.

Thus $s=u+v l$ for $u \neq 0$. We now show $v=0$, so $s \in B$. Linearizing $y \rightarrow c+\lambda d$ in (2.5) and setting $x=b$ for $b, c, d \in B$ yields

$$
\begin{aligned}
0= & Q([b t, u+v l, c],[b, t,(u+v l)(d l)])+Q([b t, u+v l, d l],[b, t,(u+v l) c]) \\
= & Q(-[b t, c, v l],[b, t,(d u) l])+Q([b t, u, d l]+[b t, v l, d l],[b, t,(v \bar{c}) l]) \\
= & \quad(\operatorname{since}[B, B, B]=0) \\
= & \quad(v[b t, c] l, d u[b, t] l)+Q(d[b t, u] l, v \bar{c}[b, t] l) \\
= & (\operatorname{since}[B, B l, B l] \subset B \text { where } Q(B, B l)=0 \text { by (1.4)) } \\
= & =(v[b t, c], d u[b, t])-\mu Q(d[b t, u], v \bar{c}[b, t])
\end{aligned}
$$


for all $d$, so nonsingularity of $Q$ and skewness of commutators gives

$$
v[b t, c][b, t] \bar{u}-v \bar{c}[b, t][b t, u]=0
$$

for all $b, c$. If $u \notin \Phi 1$ we set $c=1$ to see $v[b, t][b t, u]=0$, and since by Lemma 2.2(ii) we can cancel $[b, t]$ and $[b t, u]$ on a dense set of $b$ 's we get $v=0$. On the other hand, if $u=\alpha 1 \in \Phi 1$ (for $\alpha \neq 0$ since $u \neq 0$ ) then (*) implies $v[b t, c][b, t]=0$, and again by Lemma 2.2(ii) we can cancel $[b, t]$ and $[b t, c]$ on a dense set of $b$ 's and $c$ 's to get $v=0$.

Thus $v=0$ and $s=u \in B$. We will show $s \in \Phi$. Setting $x=b \in B$ and $y=$ $\lambda c+l$ in (2.4) for $c \in B$ and identifying coefficients of $\lambda$ yields

$$
\begin{aligned}
0 & =Q([b t, s, l],[b, t, s l] s \cdot c) \quad(\text { since } s, t, b, c \in B \text { and }[B, B, B]=0) \\
& =Q([b t, s] l, s[b, t] \bar{s} \bar{c} l)=-\mu Q([b t, s], s[b, t] \bar{s} \bar{c}) \\
& =-\mu Q([b t, s] c, s[b, t] \bar{s})=-\mu Q(c,[\overline{b t, s}] s[b, t] \bar{s}) \quad \text { by }(1.3)(\mathrm{i})
\end{aligned}
$$

so by nonsingularity of $Q$ and skewness of commutators we must have $[b t, s] s[b, t] \bar{s}$ $=0$ for all $b$. Then we can cancel $s$ and $[b, t]$ for a dense set of $b$ 's by Lemma 2.2 (ii), so $[b t, s]=0$ on a dense set and hence everywhere; then $[B, s]=0$ forces $s \in \Phi 1$ by Lemma 2.2 (ii).

2.6 REMARK. The weaker identity (2.5) is not enough to force $t$ or $s$ into $\Phi 1$, since one can show via (1.9) that it is satisfied identically for $s=2 Q(t) 1-T(t) \bar{t}$.

Our second impossibility is more difficult.

2.7 SECOND IMPOSSIBLE IDENTITY. A quaternion or octonion algebra cannot satisfy an identity of the form

$$
Q\left((y t) x, s^{-1}\{(s \bar{y})\{(t x) y-(y t) x\}\}\right)=0
$$

identically in $x$ and $y$ for invertible elements $t, s$.

Proof. If $s, t$ can be imbedded in a common quaternion subalgebra $B$ of $C$ (e.g. when $C=B$ is itself quaternion, or when $s$ or $t$ lies in $\Phi 1$ by Lemma 2.1 (ii)), this is fairly easy to see. Indeed, for $x, y, s, t$ in the associative algebra $B$ we would have

$$
\begin{aligned}
0 & =Q(y t x, \bar{y}[t x, y])=Q\left(y^{2} t x,[t x, y]\right) \quad(\text { by }(1.3)(\mathrm{i})) \\
& =T(y) Q(y t x,[t x, y])-Q(y) Q(t x,[t x, y]) \quad(\text { by }(1.3)(\mathrm{iv})) \\
& =T(y) Q(y t x,[t x, y])
\end{aligned}
$$

since $Q(a,[a, b])=Q(1,[a, b] \bar{a})=T([a, b \bar{a}])=0$. Since $T(y) \neq 0$ we can cancel it to get (replacing $x$ by $\left.t^{-1} x\right) Q(y x,[x, y])=0$ for all $x, y$ in a quaternion algebra as in (1.5)III; in particular, for $y=w \in \Omega, x=j$, we have $0=Q(w j,(\bar{w}-w) j)=$ $Q(w, \bar{w}-w) Q(j)$, hence $0=Q(w, \bar{w}-w)=Q(w, 2 w-T(w) 1)=4 Q(w)-$ $T(w)^{2}$, whereas by (1.5)II in characteristic 2 we can find $w \in \Omega$ with $T(w)=1$ and in characteristic $\neq 2$ we can find invertible skew $w=i$ with $T(w)=0 \neq Q(w)$. 
The general octonion case is more complicated. We may assume $s, t \notin \Phi 1$. Replacing $x$ by $t^{-1} x,(2.8)$ becomes

$$
\begin{aligned}
0= & Q\left((y t)\left(t^{-1} x\right), s^{-1}\{[s, \bar{y}, x y]+s \cdot \bar{y}(x y)\}\right) \\
& -Q\left(\bar{s}^{-1}\left\{(y t)\left(t^{-1} x\right)\right\},(s \bar{y})\left\{(y t)\left(t^{-1} x\right)\right\}\right) \quad(\text { by }(1.3)(\mathrm{i})) \\
= & Q\left((y t)\left(t^{-1} x\right),-s^{-1}[s, y, x y]+T(y) x y-y x y\right) \\
& -Q\left(\bar{s}^{-1}\left\{(y t)\left(t^{-1} x\right)\right\},(s \bar{y})\left\{(y t)\left(t^{-1} x\right)\right\}\right) \quad(\text { by }(1.8),(1.3)) \\
= & -Q\left((y t)\left(t^{-1} x\right), s^{-1}(y[s, y, x])\right)+T(y) Q\left((y t)\left(t^{-1} x\right), x y\right) \\
& -Q\left((y t)\left(t^{-1} x\right), Q(y, \bar{x}) y-Q(y) T(x) 1+Q(y) x\right) \\
& -Q\left(\bar{s}^{-1}, s \bar{y}\right) Q(y t) Q\left(t^{-1} x\right) \quad(\mathrm{by}(1.9),(1.3)(\mathrm{iii})) \\
= & -Q\left((y t)\left(t^{-1} x\right), s^{-1}(y[s, y, x])\right)+T(y) Q\left((y t)\left(t^{-1} x\right), x y\right) \\
& -Q(\bar{y}, x) Q\left((y t)\left(t^{-1} x\right),(y t) t^{-1}\right)+T(x) Q\left((y t)\left(t^{-1} x\right),(y t)\left(t^{-1} \bar{y}\right)\right) \\
& -Q(y) Q\left((y t)\left(t^{-1} x\right), t\left(t^{-1} x\right)\right)-T(\bar{y}) Q(y) Q(x) \quad(\mathrm{using}(1.8)) \\
= & -Q\left((y t)\left(t^{-1} x\right), s^{-1}(y[s, y, x])\right)+T(y) Q\left((y t)\left(t^{-1} x\right), x y\right) \\
& -Q(\bar{y}, x) Q(y) Q(t) Q\left(t^{-1}\right) Q(x, 1)+T(x) Q(y) Q(t) Q\left(t^{-1}\right) Q(x, \bar{y}) \\
& -Q(y) Q(y, 1) Q(t) Q\left(t^{-1}\right) Q(x)-T(y) Q(y) Q(x),
\end{aligned}
$$

so (2.8) may be rewritten as

$$
\begin{aligned}
0= & T(y)\left\{Q\left((y t)\left(t^{-1} x\right), x y\right)-2 Q(y) Q(x)\right\} \\
& -Q\left((y t)\left(t^{-1} x\right), s^{-1}(y[s, y, x])\right) .
\end{aligned}
$$

The impossibility of this is easily seen if the characteristic $\neq 2$ or if $T(s) \neq 0$ : in this case $T \neq 0$ on the subspace $\Phi 1+\Phi s$, and for $y$ in this subspace $[s, y, C]=0$, therefore (2.9) for such $y$ becomes $T(y) F(x, y)=0$, whence nonvanishing of $T(y)$ forces $F(x, y)=0$, and we would have $Q\left((y t)\left(t^{-1} x\right), x y\right)-2 Q(y) Q(x)=0$. In particular, $y=s$ would yield

$$
\begin{aligned}
2 Q(x) Q(s) & Q(t)=Q\left((s t)\left(t^{-1} x\right), x s\right) Q(t)=Q\left(s t,(x s)\left(\bar{x} \bar{t}^{-1}\right)\right) Q(t) \\
& =Q(s t,(x s)(\bar{x} t)) \quad(\text { by }(1.3)(\mathrm{ii})) \\
& =Q(s t,(x s)\{Q(x, t) 1-\bar{t} x\})=Q(x, t) Q(s t, x s)-Q(s t, x(s \bar{t}) x) \\
& \\
= & (\text { by }(1.7)(\mathrm{iii})) \\
= & Q(x, t) Q(x s, s t)-Q(s t, Q(x, t \bar{s}) x-Q(x) t \bar{s}) \quad(\text { by }(1.3)(\mathrm{iii}))
\end{aligned}
$$

We can choose $x \perp t$, st with $Q(x) \neq 0$ (since $\{t, s t\}^{\perp}$ has dimension 6 and the maximum possible dimension of a totally isotropic subspace is 4) to see $Q(s t, t \bar{s})=$ $2 Q(s) Q(t)$, and hence $0=Q(x, t) Q(x, s t \bar{s})-Q(x, t \bar{s}) Q(x, s t)$. Since $s \notin \Phi 1$ we cannot have $s t \bar{s} \in \Phi t \bar{s}$ or $t \in \Phi t \bar{s}$ (cancelling $t \vec{s}$ or $t$ ), so we can find an $x$ with $Q(x, s t \bar{s}) \neq 0, Q(x, t) \neq 0$, but $Q(x, t \bar{s})=0$, contrary to the above relation. 
Finally, assume the characteristic is 2 and $T(s)=0$. Here (2.9) becomes via (1.3)(ii)

$$
T(y) Q((y t)(\bar{t} x), x y)=Q\left((y t)(\bar{t} x), s^{-1}(y[s, y, x])\right) .
$$

By Lemma 2.1 (i) we can write $C=B+B l$ for $l=s$; let $t=u+v l, x=s=l, y=b$ for $u, v, b \in B$, so the associator on the right collapses and $\left(2.9^{\prime}\right)$ becomes

$$
\begin{aligned}
0 & =T(b) Q(b(u+v l) \cdot(\bar{u}+v l) l, l b)=T(b) Q((b u+(v b) l) \cdot(\bar{u} l+\mu v), \bar{b} l) \\
& =T(b) Q(\{\bar{u}(b u)+\mu(v b) \bar{v}\} l, \bar{b} l) \quad(\text { since } B \perp B l) \\
& =\mu T(b) Q(\bar{u} b u+\mu v b \bar{v}, \bar{b})
\end{aligned}
$$

identically in $b$. Since $T \neq 0$ on $B$ we can cancel it and use (1.3)(i) to get

$$
\begin{aligned}
0=T(\bar{u} b u b+\mu v b \bar{v} b)=T(\bar{u}\{Q(b, \bar{u}) b+Q(b) \bar{u}\}+\mu v\{Q(b, v) b+Q(b) v\}) \\
\quad(\text { by }(1.3)(\text { iii) in characteristic 2) } \\
=\{Q(b, \bar{u}) Q(b, u)+\mu Q(b, v) Q(b, \bar{v})\}+Q(b)\left\{T\left(\bar{u}^{2}\right)+\mu T\left(v^{2}\right)\right\} .
\end{aligned}
$$

Choosing $b \perp u$, $v$ with $Q(b) \neq 0$ shows $T\left(\bar{u}^{2}\right)+\mu T\left(v^{2}\right)=0$, so

$$
Q(b, u) Q(b, \bar{u})=\mu Q(b, v) Q(b, \bar{v}) .
$$

Then $b \perp v$ in (*) forces $b \perp u$ or $b \perp \bar{u}$, hence $u=\lambda v$ or $\bar{u}=\lambda v$ by nonsingularity of $Q$ on $B$; then choosing $b \not v, \bar{v}$ in (*) yields $\lambda^{2}=\mu$; but then $Q(t)=Q(u)-$ $\mu Q(v)=\lambda^{2} Q(v)-\mu Q(v)=0$ by (1.4), contrary to the invertibility of $t$.

3. The standard composition triples. We now examine the basic examples of composition triples, the composition algebras with standard triple products. A permutation of a triple product $\left\{x_{1} x_{2} x_{3}\right\}$ has the form

$$
\left\{x_{1} x_{2} x_{3}\right\}_{\pi}=\left\{x_{\pi(1)} x_{\pi(2)} x_{\pi(3)}\right\} \quad\left(\pi \in S_{3}\right) .
$$

Notice that if the original triple permits composition, so does any permutation thereof: $Q\left(\left\{x_{1} x_{2} x_{3}\right\}_{\pi}\right)=Q\left(x_{\pi(1)}\right) Q\left(x_{\pi(2)}\right) Q\left(x_{\pi(3)}\right)=Q\left(x_{1}\right) Q\left(x_{2}\right) Q\left(x_{3}\right)$. In any nonassociative algebra $A$ the left and right standard triple products are

$$
\{x y z\}_{L}=(x y) z, \quad\{x y z\}_{R}=x(y z),
$$

and the standard triple products on $A$ are the permutations of the left and right standard products. Notice that if $A$ is a composition algebra with norm $Q$, then all the standard triple products $T$ permit composition with $Q$, yielding the standard composition triples $(T, Q)$.

Thus a nonassociative algebra gives rise to 12 standard products $\{x y z\}$ given by

$$
\begin{array}{llllll}
(x y) z, & (y z) x, & (z x) y, & (y x) z, & (z y) x, & (x z) y, \\
x(y z), & y(z x), & z(x y), & y(x z), & z(y x), & x(z y) .
\end{array}
$$

In general (see 3.11) these are nonisotopic. However, if $\boldsymbol{A}$ has an anti-automorphism $J$ (e.g. an involution), then any standard product is isomorphic to its opposite (the same product taken in the opposite algebra): $(x y) z \cong z(y x)$ and $x(y z) \cong(z y) x$ by setting $F=J$ in (0.2). For a composition algebra $A$ this leads to the following classification of standard triple products. 
3.4 NONISOTOPY THEOREM. Let $A$ be a finite-dimensional composition algebra.

(i) If $A$ is commutative and associative (Types I-II), then all 12 standard products coincide with $\{x y z\}=x y z$.

(ii) If $A$ is associative but not commutative (Type III), then there are 6 distinct standard products $\{x y z\}$ consisting of 3 nonisotopic pairs

$$
x y z \cong z y x, \quad y z x \cong x z y, \quad z x y \cong y x z .
$$

(iii) If $A$ is not associative (Type IV), then there are 12 distinct standard products $\{x y z\}$ consisting of 6 nonisotopic pairs

$$
\begin{array}{lll}
(x y) z \cong z(y x), & (y z) x \cong x(z y), & (z x) y \cong y(x z), \\
x(y z) \cong(z y) x, & y(z x) \cong(x z) y, &
\end{array}
$$

PROOF. An intrinsic characterization of the various standard products can be obtained by means of the concepts of "outside" and "adjacent" in a triple product. We say $x_{i}$ is outside in a triple product $\left\{x_{1} x_{2} x_{3}\right\}$ if there is a rational map $G$ : $X \rightarrow \operatorname{End}(X)$ which "cancels" $x_{i}$, i.e.

$$
G\left(x_{i}\right)\left(\left\{x_{1} x_{2} x_{3}\right\}\right)=H\left(x_{j}, x_{k}\right) \quad(\{i, j, k\}=\{1,2,3\})
$$

for some bilinear map $H: X \times X \rightarrow X$ which is "injective" in the sense that there exists $u \in X$ such that $H(x, u)=0 \Rightarrow x=0$. Two variables $x_{i}, x_{j}(i<j)$ in $\left\{x_{1} x_{2} x_{3}\right\}$ are adjacent if there exists an invertible linear operator $H \in \operatorname{End}(X)$ and a rational map $G: X \rightarrow X$ such that

$$
M_{i j}\left(x_{i}, G\left(x_{i}\right)\right)=H
$$

where the multiplication operator $M_{i j}$ is defined by $M_{i j}\left(x_{i}, x_{j}\right)\left(x_{k}\right)=\left\{x_{1} x_{2} x_{3}\right\}$. These properties are isotopy-invariant: if $\left\{x_{1} x_{2} x_{3}\right\}^{\prime}=F_{0}^{-1}\left\{F_{1}\left(x_{1}\right) F_{2}\left(x_{2}\right) F_{3}\left(x_{3}\right)\right\}$ is an isotope as in (0.3) then $x_{i}$ will remain outside via $G^{\prime}\left(x_{i}\right)=G\left(F_{i}\left(x_{i}\right)\right) \circ F_{0}$, $H^{\prime}\left(x_{j}, x_{k}\right)=H\left(F_{j}\left(x_{j}\right), F_{k}\left(x_{k}\right)\right), u^{\prime}=F_{k}^{-1}(u)$, and $x_{i}, x_{j}$ will remain adjacent via $H^{\prime}=F_{0}^{-1} \circ H \circ F_{k}, G^{\prime}\left(x_{i}\right)=F_{j}^{-1}\left(G\left(F_{i}\left(x_{i}\right)\right)\right)$.

Note that in the standard product $(x y) z$ in an alternative algebra we have $z$ outside (via $\left.G(z)=R(z)^{-1}, H(x, y)=x y, u=1\right)$, and $x, y$ and $y, z$ are adjacent (via $H=I, G(a)=a^{-1}$ by the inverse identities (1.8)), and similarly in the product $x(y z)$ the $x$ is outside and $x, y$ and $y, z$ are adjacent.

We claim that if $A$ is not commutative then $x, z$ are not adjacent in the products $(x y) z$ and $x(y z)$. It suffices to consider the first case. Suppose $(x y) G(x)=H(y)$ for rational $G$; then $y=1$ would yield $x G(x)=H(1)=t$, so $G(x)=x^{-1} t$ is defined at $x=1$, in which case $x=1$ shows $y t=H(y)$ (in particular, $H=R(t)$ invertible implies $t$ is invertible), and adjacency reduces to $(x y)\left(x^{-1} t\right)=y t$. But then $x=t$ would yield $t y=y t$ for all $y$, and in a composition algebra this implies $t$ is a scalar, so we can cancel it to get $x y x^{-1}=y$, i.e. that $A$ is commutative.

Next, we claim that if $A$ is not commutative and associative then $y$ is not outside in the products $(x y) z$ and $x(y z)$. Again it suffices to consider the first case. Suppose $G(y)((x y) z)=H(x, z)$ for rational $G$ and bilinear injective $H$. Setting $x=y^{-1}$ yields $G(y)(z)=H\left(y^{-1}, z\right)$, so $H\left(y^{-1},(x y) z\right)=G(y)((x y) z)=H(x, z)$; replacing $y$ by $y^{-1}, x$ by $x y$ yields $H(y, x z)=H(x y, z)$ for a dense set of $x, y$, hence everywhere. Setting $y=1$ we see $H(x, z)=H(x z)$ for $H(a)=H(1, a)$, therefore 
$H(y(x z))=H((x y) z)$, i.e. $H([y, x, z])=H([y, x] z)$; in particular, $z=1$ shows $H$ vanishes on commutators, $H([y, x])=0$. Injectivity means $H(x u)=0 \Rightarrow x=0$, so alternativity $H([y, u] u)=H([y, u, u])=0$ forces $[y, u]=0$, then $H([y, u, z] u)=$ $H([y, u, u z])($ by $(1.9))=H([y, u] u z)=0$ forces $[y, u, z]=0$ too, so $u$ lies in the center of $A$; then $H([x, y] u)=H([x, y u])=0$ forces commutativity $[x, y]=0$, and $H([x, y, z] u)=H([x, y, z u])=H([x, y] z u)=0$ forces associativity $[x, y, z]=0$.

Finally, we claim that if $A$ is not associative then $x$ is not outside in the product $(x y) z$, nor $z$ in $x(y z)$. As usual if suffices to consider the former case. Suppose $G(x)((x y) z)=H(y, z)$; then $y=x^{-1}$ shows $G(x)(z)=H\left(x^{-1}, z\right)$, hence $H\left(x^{-1},(x y) z\right)=H(y, z)$, so replacing $x$ by $x^{-1}$ and $y$ by $x y$ yields $H(x, y z)=$ $H(x y, z)$ on a dense set and therefore everywhere. Setting $z=\mathrm{i}$ we see $H(x, y)=$ $H(x y)$ for $H(a)=H(a, 1)$, therefore $H(x(y z))=H((x y) z)$ and $H$ vanishes on associators. Teichmüller's Identity $x[y, z, u]+[x, y, z] u=[x, y, z u]-[x, y z, u]$ $+[x y, z, u]$ then shows $H([x, y, z] u)=-H(x[y, z, u])$. In particular, alternativity $H([x, y, u] u)=-H(x[y, u, u])=0$ forces $[x, y, u]=0$ by injectivity, hence $H([x, y, z] u)=-H(x[y, z, u])=0$ forces $[x, y, z]=0$, so $A$ must be associative.

We now apply these concepts to the proof of the theorem. (i) is clear. For (ii), if $A$ is associative but not commutative then there are 6 distinct products in (3.3), coming in 3 pairs of isomorphic products via the involution on $A$. The nonisotopy of products from different pairs follows because they have different adjacency patterns (or different outsideness patterns): in $x y z$ we have $x, y$ and $y, z$ but not $x, z$ adjacent (or $x$ and $z$ but not $y$ outside). Similarly, in (iii) if $A$ is not associative (hence not commutative either) then all 12 products (3.3) are distinct, coming via the involution in 6 pairs as in Theorem 3.4 (iii). The nonisotopy of products from different pairs follows because they have different adjacency plus outsideness patterns: $(x y) z$ has $x, y$ and $y, z$ but not $x, z$ adjacent, and $z$ but not $x$ or $y$ outside, while $x(y z)$ has the same adjacency pattern but now $x$ but not $y$ or $z$ is outside.

We now digress from composition algebras to make a few remarks about isotopy of standard triple products in general nonassociative algebras; these results will not be used in the rest of the paper.

3.7 REMARK. The above proof works in any alternative algebra $A$ with a dense set of invertible elements for which $[t, A]=0 \Rightarrow[t, A, A]=0$ (e.g. if $A$ has characteristic $\neq 3$ ).

3.8 RemarK. A different characterization can be used to distinguish $(x y) z$ and $x(y z)$ in any unital alternative algebra. We say a triple product has the isotopic left Moufang property if there exists an invertible operator $H(y)$ and a map $G(x ; y)$ on $X$ quadratic in $x$ and linear in $y$ such that

$$
L(x, y) H L(x, z)=L(G(x ; y), z) .
$$

It is easy to see this is isotopy-invariant and satisfied by $x(y z)$ in an alternative algebra $(H=I, G(x ; y)=x y x$ by (1.7)(i)), but as in (3.4) one can show $(x y) z$ satisfies (3.9) only when $A$ is associative.

3.10 REMARK. If $A$ is unital associative but not commutative, of arbitrary dimension, then the only standard triple products $x y z, z y x, y z x, x z y, z x y, y x z$ which can be isotopic are the opposite products $x y z \sim z y x, y z x \sim x z y, z x y \sim y x z$, and this 
occurs precisely when $A$ possesses an anti-automorphism. It suffices to prove $x y z$ is isotopic only to $z y x$, and this only when $A$ has an anti-automorphsm.

Throughout we suppose $F_{0}\left(x_{1} x_{2} x_{3}\right)=F_{1}\left(x_{\pi(1)}\right) F_{2}\left(x_{\pi(2)}\right) F_{3}\left(x_{\pi(3)}\right)$ as in $(0.3)$ and set $f_{i}=F_{i}(1)$ (so $f_{0}=f_{1} f_{2} f_{3}$ ). Then $F_{0}(a)=f_{1} f_{2} F_{3}(a)=F_{1}(a) f_{2} f_{3}$ shows $L\left(f_{1} f_{2}\right)=$ $F_{0} F_{3}^{-1}, R\left(f_{2} f_{3}\right)=F_{0} F_{1}^{-1}$ are invertible, hence $f_{1} f_{2}$ and $f_{2} f_{3}$ are invertible elements, so all $f_{1}, f_{2}, f_{3}$ are invertible elements. Then

$$
F_{3}(a)=f_{2}^{-1} f_{1}^{-1} F_{0}(a), \quad F_{1}(a)=F_{0}(a) f_{3}^{-1} f_{2}^{-1}, \quad F_{2}(a)=f_{1}^{-1} F_{0}(a) f_{3}^{-1}
$$

so $G(a)=F_{0}(a) f_{0}^{-1}$ has

$$
G(1)=1, f_{0}^{-1}=f_{3}^{-1} f_{2}^{-1} f_{1}^{-1}, \quad G\left(x_{1} x_{2} x_{3}\right)=G\left(x_{\pi(1)}\right) G\left(x_{\pi(2)}\right) G\left(x_{\pi(3)}\right) .
$$

For the transpositions $x y z$ and $y x z$ it suffices to show $x y z \nsim x z y$, and for the cyclic permutations $y z x, z x y$ it suffices to show $x y z \nsim y z x$. If $x y z \sim y z x$ then $G(x y)=$ $G(y) G(x), G(y z)=G(y) G(z)$ forces $A$ to be commutative, similarly $x y z \sim x z y$ forces commutativity, and if $x y z \sim z y x$ then $G(x y)=G(y) G(x)$ so $G$ is an antiautomorphism of $A$.

3.11 REMARK. The nonisotopy results of (3.10) can be extended to arbitrary unital nonassociative algebras if we impose the finiteness condition that $L(a)$ or $R(a)$ surjective implies $L(a)$ or $R(a)$ is bijective (e.g. if $A$ is finite dimensional): if $A$ is neither associative nor commutative then the only possible isotopies among the 12 standard products (3.3) are between opposites $((x y) z \sim z(y x)$, etc.) or between transpositions $(x(y z) \sim x(z y),(x y) z \sim(y x) z$, etc.) which happens only if $A$ is isotopic to its opposite.

More precisely, one can show (i) if $A$ is not associative then $x_{1}\left(x_{2} x_{3}\right) \nsim$ $\left(x_{1} x_{2}\right) x_{3}, x_{3}\left(x_{2} x_{1}\right),\left(x_{2} x_{1}\right) x_{3}, x_{3}\left(x_{1} x_{2}\right)$; (ii) if $A$ is not commutative associative then $x_{1}\left(x_{2} x_{3}\right) \nsim x_{2}\left(x_{1} x_{3}\right), x_{2}\left(x_{3} x_{1}\right),\left(x_{3} x_{1}\right) x_{2},\left(x_{1} x_{3}\right) x_{2},\left(x_{2} x_{1}\right) x_{3}, x_{3}\left(x_{1} x_{2}\right)$; (iii) $x_{1}\left(x_{2} x_{3}\right) \sim x_{1}\left(x_{3} x_{2}\right)$ iff there is an autotopy $F(x y)=G(x) H(y)$ of $A$ such that $H$ is an anti-autotopy $H(x y)=K(y) L(x)$, dually that $x_{1}\left(x_{2} x_{3}\right) \sim\left(x_{2} x_{3}\right) x_{1}$ iff there is an anti-autotopy $F(x y)=G(y) H(x)$ such that $G$ is an autotopy $G(x y)=K(x) L(y)$; (iv) $x_{1}\left(x_{2} x_{3}\right) \sim\left(x_{3} x_{2}\right) x_{1}$ iff there is an anti-autotopy $F(x y)=G(y) H(x)$ such that $G$ is also an anti-autotopy $G(x y)=K(y) L(x)$.

By right-left symmetry of the hypotheses, it suffices to work with $x_{1}\left(x_{2} x_{3}\right)$; in all cases one assumes $F_{0}\left(x_{1}\left(x_{2} x_{3}\right)\right)=F_{1}\left(x_{\pi(1)}\right) F_{2}\left(x_{\pi(2)}\right) F_{3}\left(x_{\pi(3)}\right)$ (with some distribution of parentheses on the right), and sets $f_{i}=F_{i}(1)$; if the distribution is $a(b c)$ then $F_{0}(a)=f_{1}\left(f_{2} F_{3}(a)\right)$ shows $L\left(f_{1}\right)$ is surjective and hence by hypothesis bijective, while if the distributiion is $(a b) c$ then $F_{0}(a)=\left(F_{1}(a) f_{2}\right) f_{3}$ shows $R\left(f_{3}\right)$ is surjective and hence bijective. From this one argues as in (3.10) by subsituting $x_{1}=1, x_{3}=1$ to get in (i) $F_{0}\left(x_{1}\left(x_{2} x_{3}\right)\right)=F_{0}\left(\left(x_{1} x_{2}\right) x_{3}\right)$; (ii) $=F_{0}\left(\left(x_{1} x_{3}\right) x_{2}\right)$ or $F_{0}\left(x_{2}\left(x_{1} x_{3}\right)\right)$; (iii) $F_{0}(x y)=F_{1}(x)\left\{L\left(f_{2}\right) F_{3}(y)\right\}$ and $L\left(f_{1}\right)^{-1} F_{0}(y z)=F_{2}(z) F_{3}(y)$ and dually; (iv) $F_{0}(y z)=R\left(f_{3}\right)\left\{F_{1}(z) F_{2}(y)\right\}$ and $F_{0}(x y)=\left\{R\left(f_{3}\right)^{-1} F_{0}(y)\right\} F_{3}(x)$.

4. Reduction to the unital case. We begin the study of abstract composition triples by showing each one is isotopic to a unital one, and that a unital one inhabits the same space as a unital composition algebra. We denote the left, middle, and right mulitplications as usual in a triple system by

$$
L(x, y) z=M(x, z) y=R(y, z) x=\{x y z\} .
$$


In complete analogy with the composition algebra case we have

4.2 Unital Isotope Theorem. Any finite-dimensional triple $(T, Q)$ is isotopic to a unital one $\left(T_{u}, Q_{u}\right)$ : if $Q(u) \neq 0$ then $Q_{u}(x)=Q(u)^{-1} Q(x)$ has $Q_{u}(u)=1$ and permits composition with respect to the u-unital product

$$
\{x y z\}_{u}=\left\{R\left(u_{2}, u_{3}\right)^{-1} x, M\left(u_{1}, u_{3}\right)^{-1} y, L\left(u_{1}, u_{2}\right)^{-1} z\right\}
$$

whenever $\left\{u_{1} u_{2} u_{3}\right\}=u$.

Proof. For given $u$ we can always choose $u_{i} \in X$ with $\left\{u_{1} u_{2} u_{3}\right\}=u$ (for example, we can take $u_{1}=u_{2}=u$, where by $(0.1) L(u, u)$ is a similarity $Q(L(u, u) z)=$ $Q(u)^{2} Q(z)$, therefore injective by nondegeneracy of $Q$, therefore bijective by finitedimensionality, so $L(u, u) u_{3}=u$ for some $\left.u_{3}\right)$. Since $Q\left(u_{1}\right) Q\left(u_{2}\right) Q\left(u_{3}\right)=Q(u)$ $\neq 0$ we have $Q\left(u_{i}\right) \neq 0$, hence again by $(0.1)$ the multiplications $M_{i j}=$ $R\left(u_{2}, u_{3}\right), M\left(u_{1}, u_{3}\right), L\left(u_{1}, u_{2}\right)$ are invertible similarities with ratios $Q\left(u_{i}\right) Q\left(u_{j}\right)$. Then $(0.1)$ shows $Q\left(\{x y z\}_{u}\right)=Q(u)^{-2} Q(x) Q(y) Q(z)$, therefore $Q_{u}\left(\{x y z\}_{u}\right)=$ $Q(u)^{-3} Q(x) Q(y) Q(z)=Q_{u}(x) Q_{u}(y) Q_{u}(z)$ and $Q_{u}$ permits composition. For unitality $(0.4)$ we have

$$
\begin{aligned}
\{u u z\}_{u} & =\left\{R\left(u_{2}, u_{3}\right)^{-1} u, M\left(u_{1}, u_{3}\right)^{-1} u, L\left(u_{1}, u_{2}\right)^{-1} z\right\} \\
& =\left\{u_{1}, u_{2}, L\left(u_{1}, u_{2}\right)^{-1} z\right\}=L\left(u_{1}, u_{2}\right) L\left(u_{1}, u_{2}\right)^{-1} z=z
\end{aligned}
$$

since $u=L\left(u_{1}, u_{2}\right) u_{3}=M\left(u_{1}, u_{3}\right) u_{2}=R\left(u_{2}, u_{3}\right) u_{1}$, and similarly $\{u y u\}_{u}=y$ and $\{x u u\}_{u}=x$.

Throughout the rest of this paper we will concern ourselves solely with unital composition triples. (The passage to a unital isotope is the only step in our argument that requires finite-dimensionality.) A unital composition triple necessarily has finite dimension 1, 2, 4, 8 (except for the inseparable Type $I$ in characteristic 2), since it is based on a unital composition algebra.

4.3 Associated Algebra Theorem. If $(T, Q)$ is a unital composition triple with unit $u$, then $x \cdot{ }_{u} y=\{x u y\}$ defines a unital composition algebra $C(T, u)$ with the same unit $u$ and norm $Q$. The products $x \cdot{ }_{L, u} y=\{u x y\}$ and $x \cdot{ }_{R, u} y=\{x y u\}$ also define composition algebras with the same unit $u$ and norm $Q$, and therefore are elemental isotopes or anti-isotopes of $C(T, u)$.

Proof. The formula defines a bilinear product having $u$ as unit, $u \cdot{ }_{u} y=\{u u y\}=y$ and $x \cdot{ }_{u} u=\{x u u\}=x$ by definition (0.4) of $u$ being a unit for $T$. This algebra permits composition with respect to $Q: Q\left(x \cdot{ }_{u} y\right)=Q(\{x u y\})=Q(x) Q(u) Q(y)=$ $Q(x) Q(y)$ by $(0.1),(0.5)$. The same argument applies to $x \cdot{ }_{L, u} y$ and $x \cdot_{R, u} y$, so by (1.11) these must be elemental isotopes or anti-isotopes of $C(T, u)$.

We call $C=C(T, u)$ the composition algebra associated with the composition triple $T$ and the unit $u$. We say $T$ has Type I-IV if the associated $C$ has Type I-IV of (1.5). Our main tool in investigating composition triples is linearization of the fundamental identity $(0.1)$. Such linearizations involving $Q(x, y)$ will be no help if this vanishes identically, and by (1.6) this happens as soon as $Q(x, y)$ is singular 
(namely Type I characteristic 2), so we have to use a different argument in this case. But Type $I$ is easily taken care of in general.

4.4 TheOREM. A unital composition triple of Type I is standard: $\{x y z\}=x y z$ in terms of the product in the associated composition algebra.

Proof. Either $C=\Phi u$ in characteristic $\neq 2$, or else $C=\Sigma$ is a purely inseparable field extension of exponent 1 in characteristic 2 . The first case is trivial: the product $\Phi u$ is completely determined by $\{u u u\}=u$ by unitality $(0.4)$. In the characteristic 2 case $Q(x)$ is injective $(Q(x, y)$ vanishing identically means $Q$ is additive, and nondegeneracy reduces to anisotropy of $Q$, so $Q$ has zero kernel), therefore $Q(\{x y z\})$ $=Q(x) Q(y) Q(z)=Q(x y z)$ by (4.3) implies $\{x y z\}=x y z$.

From now on we will concern ourselves with nonsingular unital composition triples $T$ of Types II-IV. We will consistently denote the given unit of $T$ and $C$ by 1 . One would expect that once a composition triple is forced to co-exist with a composition algebra of dimension $1,2,4$, or 8 , it should be a simple matter to show it is standard. We will have to work surprisingly hard to show this.

5. Reduction to the strictly unital case. Our next step is to analyze the associated left and right products to show that a unital composition triple necessarily has a permutation which is either strictly unital in the sense that the unit is "central"

$$
\{x y 1\}=\{x 1 y\}=\{1 x y\}=x y
$$

or is left t-unital

$$
\{x y 1\}=\left(x t^{-1}\right)(t y), \quad\{x 1 y\}=\{1 x y\}=x y
$$

or is right s-unital

$$
\{x y 1\}=\{x 1 y\}=x y, \quad\{1 x y\}=\left(x s^{-1}\right)(s y)
$$

for invertible nonnuclear elements $t, s$. (If $t$ or $s$ were nuclear in (5.2), (5.3) we could cancel it and have the strictly unital case (5.1).)

If $T$ is unital with associated $C$ as in (4.3), then we saw $\{x y 1\}=\left(x t^{-1}\right)(t y)$ or $\left(y t^{-1}\right)(t x),\{1 y z\}=\left(y s^{-1}\right)(s z)$ or $\left(z s^{-1}\right)(s y)$ for invertible $t, s$. This leads to 4 possible combinations;

$$
\begin{array}{lll}
\text { (A) } & \{x y 1\}=\left(x t^{-1}\right)(t y), & \{1 y z\}=\left(y s^{-1}\right)(s z), \\
\text { (B) } & \{x y 1\}=\left(x t^{-1}\right)(t y), & \{1 y z\}=\left(z s^{-1}\right)(s y), \\
\text { (C) } & \{x y 1\}=\left(y t^{-1}\right)(t x), & \{1 y z\}=\left(y s^{-1}\right)(s z), \\
\text { (D) } & \{x y 1\}=\left(y t^{-1}\right)(t x), & \{1 y z\}=\left(z s^{-1}\right)(s y) .
\end{array}
$$

We must show cases (B), (C) are permutations of case (A), that case (D) never occurs, and that in case (A) at least one of $t, s$ must be nuclear so we can cancel one or both and obtain right- $s$ or left- $t$ or strictly unital product as in 5.3, 5.2, 5.1.

We begin by reducing cases (B), (C) to case (A).

5.5 Lemma. A unital triple $\{x y z\}$ as in case (B) is a permutation of a triple $\{x y z\}^{\prime}=\{x z y\}$ of case (A); a triple $\{x y z\}$ of case (C) is a permutation of a triple $\{x y z\}^{\prime}=\{y x z\}$ of case $(\mathrm{A})$. 
Proof. If $\{x y z\}$ falls under case (B) then the triple $\{x y z\}^{\prime}=\{x z y\}$ has associated composition algebra the $t$-isotope $C^{\prime}=C^{(t)}$ of $C: x \cdot{ }^{\prime} z=x \cdot{ }_{t} z=\left(x t^{-1}\right)(t z)$ as in (1.10)(i), and

$$
\begin{aligned}
& \{x y 1\}^{\prime}=\{x 1 y\}=x y=\left(x \cdot{ }^{\prime} t\right) \cdot{ }^{\prime}\left(t^{-1} \cdot{ }^{\prime} y\right), \\
& \{x 1 z\}^{\prime}=\{x z 1\}=\left(x t^{-1}\right)(t z)=x \cdot{ }^{\prime} z, \\
& \{1 y z\}^{\prime}=\{1 z y\}=\left(y s^{-1}\right)(s z)=\left(y \cdot{ }^{\prime} s^{\prime-1}\right) \cdot{ }^{\prime}\left(s^{\prime} \cdot{ }^{\prime} z\right)
\end{aligned}
$$

for $s^{\prime}=t^{-1} \cdot{ }^{\prime} s=t^{-1} s$ (where we have used (1.10)(iii),(ii) to see $C=C^{\left(t^{-1}\right)}$ is recoverable from $C^{\prime}$, and the product $\left(y s^{-1}\right)(s z)$ is that of $C^{(s)}=\left\{C^{\left(t^{-1}\right)}\right\}^{(s)}=C^{\prime\left(s^{\prime}\right)}$ for $\left.s^{\prime}=t^{-1} \cdot{ }^{\prime} s=t^{-1} s\right)$. This shows $\{x y z\}^{\prime}$ falls under case (A).

Similarly, in case (C) the product $\{x y z\}^{\prime}=\{y x z\}$ has $C^{\prime}=C^{(s)}, C=C^{\left(s^{-1}\right)}$,

$$
\begin{aligned}
& \{x y 1\}^{\prime}=\{y x 1\}=\left(x t^{-1}\right)(t y)=\left(x \cdot{ }^{\prime} t^{\prime-1}\right) \cdot{ }^{\prime}\left(t^{\prime} \cdot{ }^{\prime} y\right), \\
& \{x 1 z\}^{\prime}=\{1 x z\}=\left(x s^{-1}\right)(s z)=x \cdot{ }^{\prime} z, \\
& \{1 y z\}^{\prime}=\{y 1 z\}=y z=\left(y \cdot{ }^{\prime} s\right) \cdot{ }^{\prime}\left(s^{-1} \cdot{ }^{\prime} z\right)
\end{aligned}
$$

for $t^{\prime}=s^{-1} \cdot{ }^{\prime} t=s^{-1} t$, and therefore falls under case (A).

Next we get rid of case (D). If $C$ is commutative (Type I or II) then all cases reduce to case (A), so case (D) never occurs as a distinct case. If $C$ is noncommutative (Type III or IV) then case (D) never appears at all.

5.6 LEMMA. There are no unital composition triples of Type III or IV falling under case (D) in (5.4).

Proof. By (D), linearizing $x \rightarrow x, 1$ and $z \rightarrow z, 1$ in (0.1) yields

(i) $Q\left(\{x y z\},\left(z s^{-1}\right)(s y)\right)=T(x) Q(y) Q(z \circ)$,

(ii) $Q\left(\{x y z\},\left(y t^{-1}\right)(t x)\right)=Q(x) Q(y) T(z)$

and further linearizing $y, z$ to 1 in the first of these yields (using (1.8))

(i) $Q(\{x y z\}, z)=T(x) T(y) Q(z)-Q\left(x z,\left(z s^{-1}\right)(s y)\right)$,

(ii) $Q(\{x y z\}, y)=T(x) Q(y) T(z)-Q\left(\left(y t^{-1}\right)(t x),\left(z s^{-1}\right)(s y)\right)$.

Since these hold identically we deduce

$$
\begin{aligned}
0= & Q(y) T(y) Q(\bar{t} x)-Q\left(\{\bar{t} x, y, y\},\left(y t^{-1}\right)(t(\bar{t} x))\right) \quad(\text { by }(\mathrm{D} 1)(\mathrm{ii})) \\
= & Q\left(y^{2}, y\right) Q(\bar{t}) Q(x)-Q(\{\bar{t} x, y, y\},(y \bar{t}) x) \quad(\text { by }(0,1),(1.3)(\mathrm{ii})) \\
= & Q\left(\left(y^{2} \bar{t}\right) x,(y \bar{t}) x\right)+Q(\{\bar{t} x,(y \bar{t}) x, y\}, y)-T(\bar{t} x) Q(y,(y \bar{t}) x) T(y) \\
& +Q\left(\left(y t^{-1}\right)(t(\bar{t} x)),\left(y s^{-1}\right)(s\{(y \bar{t}) x\})\right) \\
& +Q\left(\left\{((y \bar{t}) x) t^{-1}\right\}\{t(\bar{t} x)\},\left(y s^{-1}\right)(s y)\right)
\end{aligned}
$$

(linearizing $y \rightarrow y,(y \bar{t}) x$ in (D.2)(ii)) 


$$
\begin{aligned}
= & Q\left(\left(y^{2} \bar{t}\right) x,(y \bar{t}) x\right)+[T(\bar{t} x) T((y \bar{t}) x) Q(y) \\
& \left.-Q\left((\bar{t} x) y,\left(y s^{-1}\right)(s\{(y \bar{t}) x\})\right)\right] \\
& -T(\bar{t} x) Q((y \bar{t}) x, T(y) y)+Q\left((y \bar{t}) x,\left(y s^{-1}\right)(s\{(y \bar{t}) x\})\right) \\
& +Q\left(\{((y \bar{t}) x) \bar{t}\} x, y^{2}\right) \quad(\text { by }(\mathrm{D} 2)(\mathrm{i}),(1.3)(\mathrm{ii}),(1.7)(\mathrm{iii})) \\
= & Q\left((y \bar{t}) x,\left(y^{2} \bar{t}\right) x\right)+Q\left((y \bar{t}) x,\left(y^{2} \bar{x}\right) t\right) \\
& +T(\bar{t} x) Q((y \bar{t}) x, Q(y) 1-T(y) y) \\
& +Q\left(\bar{s}\left\{\left(\bar{s}^{-1} \bar{y}\right)[(y \bar{t}) x-(\bar{t} x) y]\right\},(y \bar{t}) x\right) \quad(\text { by }(1.3)(\mathrm{i})) \\
= & Q\left((y \bar{t}) x, y^{2} Q(t, x)\right)-Q(t, x) Q\left((y \bar{t}) x, y^{2}\right) \\
& +Q\left((y \bar{t}) x, \bar{s}\left\{\left(\bar{s}^{-1} \bar{y}\right)[(y \bar{t}) x-(\bar{t} x) y]\right\}\right) \\
\quad \quad(\text { linearizing }(z \bar{x}) x=Q(x) z \text { and using (1.3)(iv)) } & \\
= & Q\left((y \bar{t}) x, s^{-1}\{(s \bar{y})[(y \bar{t}) x-(\bar{t} x) y]\}\right) \quad(\text { by }(1.3)(\mathrm{ii}))
\end{aligned}
$$

identically, which is impossible in Types III and IV by Second Impossibility 2.7

Finally, we must show that in case (A) one of $t, s$ is forced to be nuclear. Case (A) products such as $\left\{\left(x t^{-1}\right)(t y)\right\} z$ and $x\left\{\left(y x^{-1}\right)(s z)\right\}$ exist having one nonnuclear factor, but products like $\left\{\left[\left(x t^{-1}\right)(t y)\right] s^{-1}\right\}\{s z\}$ which seem to have two nonnuclear factors do not have $\{x 1 z\}=x z$ (and when we reformulate them in terms of $x \cdot z=\{x 1 z\}=\left(x s^{-1}\right)(s z)$ we see $s$ disappears $)$.

5.7 Lemma. A unital composition triple falling under case (A) in (5.4) must have either $t$ or $s$ in the nucleus of $C$.

Proof. Nuclearity of $t, s$ is trivial if $C$ is associative, so we may assume $C$ in (4.3) is octonion. By (A), linearizing $x$ or $y$ to 1 in $(0.1)$ yields

(i) $Q\left(\{x y z\},\left(y s^{-1}\right)(s z)\right)=T(x) Q(y) Q(z)$,

(ii) $Q(\{x y z\}, x z)=Q(x) T(y) Q(z)$

and further linearization yields

(i) $Q(\{x y z\}, z)=T(x) T(y) Q(z)-Q\left(x z,\left(y s^{-1}\right)(s z)\right)$,

$$
\begin{aligned}
& \text { (ii) } Q(\{x y z\}, y)=T(x) Q(y) T(z)-Q\left(\left(x t^{-1}\right)(t y),\left(y s^{-1}\right)(s z)\right) \text {, } \\
& \text { (iii) } Q(\{x y z\}, x)=Q(x) T(y) T(z)-Q\left(\left(x t^{-1}\right)(t y), x z\right) .
\end{aligned}
$$

From these we use nonsingularity of $Q$ to see

(i) $\{x y y\}=x y^{2}-s^{-1}\left\{(s \bar{y})\left[x t^{-1}, t, y\right]\right\}$,

(ii) $\{x x y\}=x^{2} y+\left\{\left[x, s^{-1}, s y\right](\bar{x} \bar{t})\right\} \bar{t}^{-1}$ 
hold identically. For (A3)(i),

$$
\begin{aligned}
& Q\left(\{x y y\}-x y^{2}+s^{-1}\left\{(s \bar{y})\left[x t^{-1}, t, y\right]\right\}, w\right) \\
&=[-Q(\{x y w\}, y)+T(x) T(y) Q(y, w) \\
&\left.\quad-Q\left(x w,\left(y s^{-1}\right)(s y)\right)-Q\left(x y,\left(y s^{-1}\right)(s w)\right)\right] \\
&-Q\left(y^{2}, \bar{x} w\right)+Q\left(\left[x t^{-1}, t, y\right],(y \bar{s})\left(\bar{s}^{-1} w\right)\right) \\
& \quad(\text { using }(1.3)(\mathrm{i}) \text { and linearizing } z \rightarrow y, w \text { in }(\mathrm{A} 2)(\mathrm{i})) \\
&=-\left[T(x) Q(y) T(w)-Q\left(\left(x t^{-1}\right)(t y),\left(y s^{-1}\right)(s w)\right)\right]+T(x) T(y) Q(y, w) \\
&-Q\left(y^{2}, x w\right)-Q\left(x y,\left(y s^{-1}\right)(s w)\right)-Q\left(y^{2}, \bar{x} w\right) \\
&+Q\left(x y-\left(x t^{-1}\right)(t y),\left(y s^{-1}\right)(s w)\right) \quad(\text { by }(\mathrm{A} 2)(\mathrm{ii}),(1.8),(1.3)(\mathrm{ii})) \\
&=-T(x) Q(y) T(w)+T(x) T(y) Q(y, w)-Q\left(y^{2}, T(x) w\right) \quad(\text { by }(1.3)) \\
&= T(x) Q\left(-Q(y) 1+T(y) y-y^{2}, w\right) \\
&= 0 \quad(\text { by }(1.3)(\mathrm{iv})) .
\end{aligned}
$$

Dually, for (A3)(ii)

$$
\begin{aligned}
& Q\left(\{x x y\}-x^{2} y-\left\{\left[x, s^{-1}, s y\right](\bar{x} \bar{t})\right\} \bar{t}^{-1}, w\right) \\
&=[-Q(\{w x y\}, x)+Q(x, w) T(x) T(y) \\
&\left.-Q\left(\left(x t^{-1}\right)(t x), w y\right)-Q\left(\left(w t^{-1}\right)(t x), x y\right)\right] \\
&-Q\left(x^{2}, w \bar{y}\right)-Q\left(\left(x s^{-1}\right)(s y)-x y,\left(w t^{-1}\right)(t x)\right) \\
& \quad(\text { using }(1.3)(\mathrm{i}) \text { and linearizing } x \rightarrow x, w \text { in }(\mathrm{A} 2)(\mathrm{iii})) \\
&=-\left[T(w) T(y) Q(x)-Q\left(\left(w t^{-1}\right)(t x),\left(x s^{-1}\right)(s y)\right)\right]+Q(x, w) T(x) T(y) \\
& 1-Q\left(x^{2}, w y\right)-Q\left(x^{2}, w \bar{y}\right)-Q\left(\left(x s^{-1}\right)(s y),\left(w t^{-1}\right)(t x)\right) \quad(\mathrm{by}(\mathrm{A} 2)(\mathrm{ii})) \\
&=-T(w) T(y) Q(x)+Q(x, w) T(x) T(y)-Q\left(x^{2}, w T(y)\right) \\
&= T(y) Q\left(-Q(x) 1+T(x) x-x^{2}, w\right) \\
&= 0 \quad(\text { by }(1.3)(\mathrm{iv})) .
\end{aligned}
$$

Now from (0.1) and (4.3) we have

$$
\begin{aligned}
& Q\left(\{x y y\}-x y^{2},\{x x y\}-x^{2} y\right) \\
&= Q(\{x y y\},\{x x y\})-Q\left(\{x y y\}, x^{2} y\right)-Q\left(x y^{2},\{x x y\}\right)+Q(x y y, x x y) \\
&= Q(x) Q(y) Q(x, y)-T(x) Q(\{x y y\}, x y)+Q(x) Q(\{x y y\}, y) \\
&-T(y) Q(x y,\{x x y\})+Q(y) Q(x,\{x x y\})+Q(x) Q(y, x) Q(y)
\end{aligned}
$$

(linearizing (0.1) and using (1.3)(iv)) 


$$
\begin{aligned}
= & 2 Q(x) Q(y) Q(x, y)-T(x)[Q(x) T(y) Q(y)] \\
& +Q(x)\left[T(x) T(y) Q(y)-Q\left(x y,\left(y s^{-1}\right)(s y)\right)\right] \\
& -T(y)[Q(x) T(x) Q(y)]+Q(y)\left[Q(x) T(x) T(y)-Q\left(\left(x t^{-1}\right)(t x), x y\right)\right] \\
& \quad(\text { by (A1)(ii), (A2)(i),(A2)(iii)) } \\
= & 2 Q(x) Q(y) Q(x, y)-Q(x) Q(x y, y y)-Q(y) Q(x x, x y) \quad(\text { by }(1.7)(i i i)) \\
= & 2 Q(x) Q(y) Q(x, y)-Q(x) Q(x, y) Q(y)-Q(y) Q(x) Q(x, y) \\
= & 0 .
\end{aligned}
$$

By (A3) this implies

$$
Q\left(s^{-1}\left\{(s \bar{y})\left[x t^{-1}, t, y\right]\right\},\left\{\left[x, s^{-1}, s y\right](\bar{x} \bar{t})\right\} \bar{t}^{-1}\right)=0 .
$$

From $Q(a, b)=Q(\bar{a}, \bar{b})$ and skewness of associators we obtain

$$
Q\left(t^{-1}\left\{(t x)\left[x, s^{-1}, s y\right]\right\},\left\{\left[x t^{-1}, t, y\right](y \bar{s})\right\} \bar{s}^{-1}\right)=0 .
$$

Replacing $x$ by $x t, y$ by $s^{-1} y$, and using (1.7),(1.8), we get

$$
Q\left(x\left\{t\left[x t, s^{-1}, y\right]\right\},\left\{\left[x, t, s^{-1} y\right] \bar{s}\right\} Q(s)^{-1} y\right)=0 .
$$

Linearizing $x \rightarrow x+\lambda t^{-1}$ and equating coefficients of $\lambda$ yields (noting $[1, C, C]=$ $\left.\left[t^{-1}, t, C\right]=0\right)$

$$
Q\left(\left[x t, s^{-1}, y\right],\left\{\left[x, t, s^{-1}\right] s^{-1} y\right\} y\right)=0
$$

identically (using (1.3)(ii) and (1.8) as well). But this forces $t$ or $s$ in the nucleus of the octonion algebra $C$ by First Impossibility 2.3 .

Summarizing the results of 5.4-5.7, we have

5.8 THEOREM. Any unital composition triple is a permutation of a composition triple which is strictly unital, left t-unital, or right s-unital.

It remains to analyze these 3 kinds of triples. We begin with the strictly unital ones.

6. The strictly unital case. Just as the unital composition algebras have a simple structure, so do the strictly unital composition triples.

6.1 Strictly Unital Theorem. A strictly unital composition triple is left or right standard; $\{x y z\}$ is $\{x y z\}_{L}=(x y) z$ or $\{x y z\}_{R}=x(y z)$.

Proof. By (4.4) we may assume $C$ is of Type II-IV. Linearizing respectively $x, y, z$ to 1 in $(0.1)$ yields $Q(\{x y z\},\{1 y z\})=T(x) Q(y) Q(z), Q(\{x y z\},\{x 1 z\})=$ $Q(x) T(y) Q(z), Q(\{x y z\},\{x y 1\})=Q(x) Q(y) T(z)$. By strict unitality (5.1) the triple products involving 1 are $y z, x z, x y$ respectively. Note further that $T(x) Q(y) Q(z)=$ $Q((x y) z,(1 y) z)=Q(x(y z), 1(y z)) \quad$ (using $(0.1)$ and peeling off factors until we get to $Q(x, 1)=T(x))$. Similarly for the other terms on the right, so

$$
\begin{aligned}
& Q(\{x y z\}, y z)=Q((x y) z, y z)=Q(x(y z), y z), \\
& Q(\{x y z\}, x z)=Q((x y) z, x z)=Q(x(y z), x z), \\
& Q(\{x y z\}, x y)=Q((x y) z, x y)=Q(x(y z), x y) .
\end{aligned}
$$


If we introduce the "error terms"

$$
\begin{aligned}
& E_{L}(x, y, z)=\{x y z\}-\{x y z\}_{L}=\{x y z\}-(x y) z \\
& E_{R}(x, y, z)=\{x y z\}-\{x y z\}_{R}=\{x y z-x(y z)
\end{aligned}
$$

then our unitality hypothesis (5.1) can be expressed by saying that $E_{L}, E_{R}$ vanish if one of the variables is replaced by 1 , while (6.2) can be expressed by

$$
\begin{aligned}
Q(E(x, y, z), y z) & =Q(E(x, y, z), x z) \\
& =Q(E(x, y, z), x y)=0 \quad\left(E=E_{L}, E_{R}\right) .
\end{aligned}
$$

Such functions $E$ have a very special form.

6.4 KeY LEMMA. Let $E$ be a trilinear function on a unital nonsingular composition algebra C of Type II-IV, which vanishes whenever one of the variables is replaced by 1 , and such that 2 of the 3 identities

$$
Q(E(x, y, z), y z)=0, \quad Q(E(x, y, z), x z)=0, \quad Q(E(x, y, z), x y)=0
$$

hold. Then all 3 identities hold, and $E(x, y, z)$ and $Q(E(x, y, z), w)$ are both alternating functions of their variables which vanish if any variable is replaced by 1 . If $C$ is associative (Types II-III) then $E$ vanishes identically.

If $C=B+B$ l is octonion (Type IV) then we have the further identities

$$
\begin{gathered}
E(x, y, x y)=E(x, y, y x)=0, \\
E(x, y, y z)=E(x, y, z) y=\bar{y} E(x, y, z), \\
E(x, y, z y)=y E(x, y, z)=E(x, y, z) \bar{y}, \\
E(x, y,(x y) z)=(E(x, y, z) x) y
\end{gathered}
$$

and $E$ is completely determined by the element $e=E(u, v, l)=w l$ where $1, u, v, u v$ is any basis for $B$ of invertible elements such that $T(u) T(v)=0$, and where $w \in B$ is orthogonal to $1, u, v$ :

(i) $E(B, B, B)=0$,

(ii) $E(B, B, B l)=E(u, v, B l)=e B=(w B) l$,

(iii) $E(B, B l, B l) \subset(e B)(B l)=B w B$,

(iv) $E(B l, B l, B l) \subset\{(e B)(B l)\}(B l)=(B w B) l$

so that $e=0$ or $w=0$ implies $E=0$. Furthermore, $e$ and $E$ will vanish as soon as $Q(e,(u v) l)=0$, which will be the case if

$$
Q\left(E(x, y, z),\left(x u^{-1}\right)(u y)\right)=0 \text { for all } x, y \in C .
$$

Proof. The hypothesis that 2 of the 3 expressions (6.5) vanish guarantees that all 3 variables $x, y, z$ appear in products $p$ in terms $Q(E(x, y, z), p)=0$; since these are quadratic, we can linearize each variable to 1 in turn and use the hypothesis that $E(x, y, z)$ vanishes if $x, y$ or $z$ is replaced by 1 , to see

$$
Q(E(x, y, z), x)=Q(E(x, y, z), y)=Q(E(x, y, z), z)=0 .
$$

This says that $Q(E(x, y, z), w)$ alternates in the 1st and 4th, 2nd and 4th, and 3rd and 4th variables; but then it alternates in all variables. In particular, holding $w$ 
fixed and using nonsingularity of $Q$ we see $E(x, y, z)$ itself is alternating in all variables. Once $E$ alternates, as soon as any 1 of the 3 identities (6.5) holds then all 3 do. Since $Q(E(x, y, z), w)$ vanishes if any of the first 3 variables is replaced by 1 , the same must be true if the 4th variable is replaced by 1 .

If $C$ is associative of Type II or III (dimension 2 or 4) then $Q(E(x, y, z), w)$ is an alternating function of 4 variables supported on a space $1^{\perp}$ of dimension 1 or 3 , hence vanishes identically. Because $Q$ is nonsingular, once $Q(E(x, y, z), w)=0$ for all $w$ we must have $E(x, y, z)=0$.

If $C$ is octonion then $E$ need not vanish (an example is the associator $E(x, y, z)=$ $\left.\{x y z\}_{L}-\{x y z\}_{R}\right)$. (6.6) follows via nonsingularity from $Q(E(x, y, x y), w)=$ $-Q(E(x, y, w), x y)=0$ (by (6.5)) and dually. To get the first bumping formula (6.7), use nonsingularity and $Q(\bar{y} E(x, y, z)-E(x, y, y z), w)=Q(E(x, y, z), y w)$ $+Q(E(x, y, w), y z)$ (by (1.3)(i) and alternation) $=0$ (linearizing $z \rightarrow z, w$ in (6.5)); since $Q(E(x, y, z), w)$ vanishes for $w=1$ we see all $E(x, y, z)$ are skew, hence applying the involution to $\bar{y} E(x, y, z)=E(x, y, y z)$ yields $E(x, y, z) y=$ $E(x, y, y z)$. The bumping formulas for $z y$ follow from those for $y z$ by passing to the opposite algebra $C^{\mathrm{op}}$ (note that (6.5) continues to hold in $C^{\text {op }}$ by alternation of $E$ ). For (6.8) we have

$$
\begin{aligned}
\{E(x, y, z) x\} y- & E(x, y,(x y) z)=\{E(x, y, z) x\}\{T(y) 1-\bar{y}\} \\
+ & E(x, z,(x y) y)+E(x y, y, x z)+E(x y, z, x y) \\
= & \quad \text { (linearizing } x \rightarrow x, x y \text { and } y \rightarrow y, z \text { in }(6.6)) \\
& +E(x, y) E(x, z, y) x-E(x, y, x z) \bar{y}+E\left(x, z, x y^{2}\right) \\
= & -T(y) E(x, z, x y)+E(x, z, T(y) x y-\underset{g}{Q(y) x)} \\
= & \quad \text { (by }(6.7) \text { and alternation) }
\end{aligned}
$$

By alternation and (6.5) we know $e=E(u, v, l)$ is orthogonal to $1, u, v, l, u v, u l, v l$; then $e \perp B$, so $e=w l \in B l$ where $w l \perp l, u l, v l$ implies $w \perp$ $1, u, v$. For (6.9)(i), by alternation and vanishing of $E$ on $1, E(B, B, B)$ is spanned by $E(u, v, u v)$, which vanishes by (6.6). For (6.9)(ii) we note by alternation and vanishing at 1 that $E(B, B, B l)$ is spanned by $E(u, v, B l), E(u, u v, B l)=$ $E(u, v, u(B l))$ (by $(6.7)$ twice $)=E(u, v,(B u) l)=E(u, v, B l)$ (by the assumed invertibility of $u)$, similarly $E(u v, v, B l)=E(u, v,(B l) v)=E(u, v, B l)$. Here $E(u, v, B l)$ is spanned by $E(u, v, l)=e, E(u, v, u l)=E(u, v, l) u=$ $e u, E(u, v, v l)=e v, E(u, v,(u v) l)=\{E(u, v, l) u\} v=(e u) v=e(v u)$ (by (6.7), (6.8), and $e=w l)$. Thus $E(u, v, B l)=e(\Phi 1+\Phi u+\Phi v+\Phi v u)=e B$. For (6.9)(iii),(iv) note that we can reduce a factor $B l$ to $B$ via $E(x, b l, B l)=$ $E(x, b l,(b \bar{B}) l)=E(x, b l,(b l) B)=E(x, b l, B)(b l)($ by $(6.7)) \subset E(x, b l, B)(B l)$ for the invertible basis elements $b \in B$, hence for all $b$. This finishes (6.9).

We know $e=w l$ is orthogonal to all the basis vectors for $C$ except possibly ( $u v) l$, so $Q(e,(u v) l)=0 \Rightarrow Q(e, C)=0 \Rightarrow e=0$ by nonsingularity. We must show 
(6.10) forces $Q(E(u, v, l),(u v) l)=0$. In characteristic $\neq 2$ the proof is easy. Linearizing $x \rightarrow x u, u$ in (6.10) and setting $z=x$ gives

$$
\begin{aligned}
0 & =Q(E(x u, y, x), u y)+Q(E(u, y, x), x(u y)) \\
& =Q(\bar{x} E(u, y, x), u y)+Q(E(u, y, x), x(u y)) \quad \text { (by }(6.7)) \\
& =2 Q(E(u, y, x), x(u y)) \quad(\text { by }(1.3)(\mathrm{i})),
\end{aligned}
$$

so setting $x=v, y=l$ shows $0=2 Q(E(u, l, v), v(u l))=-2 Q(E(u, v, l),(u v) l)$.

To establish $Q(e,(u v) l)=0$ for arbitrary characteristic we set $x=b u, y=l, z=c$ in (6.10) for $b, c \in B$ to get $Q(E(b u, l, c), b(u l))=0$. By linearized (6.7) this implies

$$
\begin{aligned}
0= & Q(-E(l u, b, c)+\bar{b} E(u, l, c)+\bar{l} E(u, b, c), b(u l)) \\
= & -Q(E(\bar{u} l, b, c), b(u l))+Q\left(E(u, l, c), b^{2}(u l)\right)+0 \\
& \quad(\text { by }(1.3)(\mathrm{i}),(1.7)(\mathrm{i}), \text { and }(6.9)(\mathrm{i}) \text { since } u, b, c \in B) \\
= & -T(u) Q(E(l, b, c), b(u l))+Q(E(u l, b, c), b(u l)) \\
& +T(b) Q(E(u, l, c), b(u l))-Q(b) Q(E(u, l, c), u l) \quad(\text { by }(1.3)) \\
= & -T(u) Q(E(l, b, c), b(u l))+0+T(b) Q(E(u, l, c), b(u l))-0
\end{aligned}
$$

(by (6.5) and the alternation of $E$ ). If $T(u)=0$ then $T(b) Q(E(u, c, l), b(u l))=0$ for all $b, c \in B$, hence $T \neq 0$ on $B$ forces $Q(E(u, c, l), b(u l))=0$, whence $c=b=v$ yields $Q(e,(u v) l)=0$. If on the other hand $T(u) \neq 0$ then by hypothesis $T(u) T(v)$ $=0$ we must have $T(v)=0$, so setting $b=v, c=u$ yields $T(u) Q(E(l, v, u), v(u l))$ $=0$, and once more $Q(e,(u v) l)=0$. Thus $Q(e,(u v) l)=0$ in all cases. This completes the proof of the lemma.

Returning to the proof of Theorem 6.1, we see that if $C$ is associative then $E_{L}=0$ and the strictly unital triple $T$ is standard: $\{x y z\}=\{x y z\}_{L}=x y z$. If $C$ is octonion we expect one of $E_{L}$ or $E_{R}$ to vanish, but we cannot predict which one; we will show that the "product" of $E_{L}$ and $E_{R}$ vanishes, forcing one of the "factors" to vanish. It suffices to establish this in some scalar extension, so we can assume $|\Phi|>2$. In this case we can by (1.5) choose a basis $1, u, v, u v$ for $B$ with $u, v$ invertible, $T(v)=0$, and $\{1, u, v\}^{\perp}=\Phi w$ for invertible $w$ (namely $u=i, v=j, w=i j$ in characteristic $\neq 2$, and in characteristic $2 u=\alpha 1+\omega, v=w=j$ where $\alpha^{2}+\alpha \neq Q(\omega)$, using $|\Phi|>2)$. By Lemma $6.4 E_{L}, E_{R}$ are completely determined by $e_{L}=w_{L} l, e_{R}=w_{R} l$ for $w_{L}=\alpha_{L} w, w_{R}=\alpha_{R} w$ in the 1-dimensional space $\Phi w=\{1, u, v\}^{\perp}$. Now

$$
\begin{aligned}
\Phi \alpha_{L} \alpha_{R} & =\alpha_{L} \alpha_{R} \mu Q(w) Q(B, B) \quad(\text { since } Q(w) \neq 0 \text { and } Q(B, B)=\Phi) \\
& =\alpha_{L} \alpha_{R} Q((w l) B,(w l) B)=Q\left(e_{L} B, e_{R} B\right)=Q\left(e_{L},\left(e_{R} B\right) \bar{B}\right)
\end{aligned}
$$

$$
=Q\left(e_{L}, e_{R} B\right)=Q\left(E_{L}(u, v, l), E_{R}(u, v, B l)\right) \quad(\text { by }(6.9)(\mathrm{ii})),
$$

so if we can prove

$$
Q\left(E_{L}(u, v, l), E_{R}(u, v, B l)\right)=0
$$


then $\alpha_{L} \alpha_{R}=0$, one of $\alpha_{L}$ or $\alpha_{R}$ is 0 , so one of $E_{L}$ or $E_{R}$ is 0 as desired. But for any $z \in C$ we have

$$
\begin{aligned}
& Q\left(E_{L}(u, v, l), E_{R}(u, v, z)\right)=Q(\{u v l\}-(u v) l,\{u v z\}-u(v z)) \\
&= Q(\{u v l\},\{u v z\})-Q(\{u v l\}, u(v z))-Q((u v) l,\{u v z\})+Q((u v) l, u(v z)) \\
&= Q(u) Q(v) Q(l, z)-Q\left(E_{L}(u, v, l), u(v z)\right)-Q\left((u v) l, E_{L}(u, v, z)\right) \\
&-Q((u v) l,(u v) z) \quad(\text { by linearized }(0.1)) \\
&= Q(u v) Q(l, z)+Q\left(E_{L}(u, v, u(v z)), l\right)-Q\left(E_{L}(u, v, z),(u v) l\right) \\
&-Q(u v) Q(l, z) \quad\left(\text { by alternation of } E_{L}\right) \\
&= Q\left(\left(E_{L}(u, v, z) v\right) u, l\right)-Q\left(E_{L}(u, v, z),(u v) l\right) \quad(\text { by }(6.7)) \\
&= Q\left(E_{L}(u, v, z),(l \bar{u}) \bar{v}-(u v) l\right) \quad(\text { by }(1.3)(\mathrm{i})) \\
&= 0 \quad(\text { for } u, v \in B) .
\end{aligned}
$$

Thus (*) holds, and either $E_{L}=0$ (so $\left.\{x y z\}=\{x y z\}_{L}\right)$ or $E_{R}=0$ (so $\{x y z\}=$ $\left.\{x y z\}_{R}\right)$.

7. The left- $t$-unital and right-s-unital cases. It remains to analyze the left- $t$ and right-s unital cases (5.2) and (5.3) for invertible nonnuclear elements $t, s$. The archetypal products of these forms are $\left\{\left(x t^{-1}\right)(t y)\right\} z$ and $x\left\{\left(y s^{-1}\right)(s z)\right\}$; these are not permutations of strictly unital triples, rather they are isotopes of the strictly unital triples $(x y) z$ and $x(y z)$. In the left- $t$-unital case the natural isotopy $\{x y z\}^{\prime}=$ $\left\{x t, t^{-1} y, z\right\}$ restores left unitality but destroys middle and right unitality. Rather than find another isotopy, or prove the natural one does indeed produce a strictly unital triple, it turns out to be just as quick to carry out a classification similar to that of 6.1 directly. Note that we do not have to hesitate between left or right standardness: only the left association is possible for $t$, and only the right association for $s$.

7.1 Left-Right Unital Theorem. A left t-unital composition triple (for nonnuclear $t$ ) is left $t$-standard,

$$
\{x y z\}=\left[\left(x t^{-1}\right)(t y)\right] z,
$$

and a right s-unital triple is right s-standard,

$$
\{x y z\}=x\left[\left(y s^{-1}\right)(s z)\right] .
$$

Thus both are isotopic to left or right standard products.

Proof. Nonnuclearity of $t, s$ means $C$ is octonion in 1.5. We carry out the proof for the left $t$-unital case, that for the right $s$-unital case being dual (apply the $t$-case to the dual triple $\left.\{x y z\}^{*}=\{\overline{\bar{z} \bar{y} \bar{x}}\}\right)$. We must show the error term

$$
E_{t}(x, y, z)=\{x y z\}-\left[\left(x t^{-1}\right)(t y)\right] z
$$


vanishes identically. As in (6.1) we linearize the basic relation (0.1) and use left $t$-unitality (5.2) to get $Q\left(\{x y z\},\left(x t^{-1}\right)(t y)\right)=Q(x) Q(y) T(z), Q(\{x y z\}, x z)=$ $Q(x) T(y) Q(z), Q(\{x y z\}, y z)=T(x) Q(y) Q(z)$. Here

$$
\begin{gathered}
Q(x) Q(y) T(z)=Q\left(x t^{-1}\right) Q(t y) T(z)=Q\left(\left\{\left(x t^{-1}\right)(t y)\right\} z,\left(x t^{-1}\right)(t y)\right), \\
\begin{aligned}
Q(x) T(y) Q(z) & =Q\left(x t^{-1}\right) Q(t) Q(y, 1) Q(z)=Q\left(x t^{-1}\right) Q(t y, t) Q(z) \\
& =Q\left(\left\{\left(x t^{-1}\right)(t y)\right\} z,\left\{\left(x t^{-1}\right) t\right\} z\right)=Q\left(\left\{\left(x t^{-1}\right)(t y)\right\} z, x z\right)
\end{aligned}
\end{gathered}
$$

(by (1.8)), similarly

$$
T(x) Q(y) Q(z)=Q\left(\left\{\left(x t^{-1}\right)(t y)\right\} z, y z\right),
$$

so by (7.2) we have

(i) $Q\left(E_{t}(x, y, z),\left(x t^{-1}\right)(t y)\right)=0$,

(ii) $Q\left(E_{t}(x, y, z), x z\right)=0$,

(iii) $Q\left(E_{t}(x, y, z), y z\right)=0$.

The last two of these show 2 of the 3 identities (6.5) hold; furthermore $E_{t}$ vanishes if any variable is set equal to 1 by left $t$-unitality (5.2) and by $(1.8)\left(E_{t}(x, y, 1)=\{x y 1\}\right.$ $\left.-\left(x t^{-1}\right)(t y)=0, E_{t}(x, 1, z)=\{x 1 z\}-x z=0, E_{t}(1, y, z)=\{1 y z\}-y z=0\right)$. Therefore we may apply Key Lemma 6.4 to $E=E_{t}$. Moreover, since $t \notin \Phi 1$ is invertible we can by (2.1)(ii) imbed it in a quaternion subalgebra, $C=B+B l$ where $B$ has basis $1, t, v, t v$ with $v$ invertible, $T(v)=0$. By (6.10) with $t=u, E_{t}$ will vanish as soon as $Q\left(E_{t}(x, y, z),\left(x t^{-1}\right)(t y)\right)=0$, and this holds by (7.3)(i). Thus $E_{t}=0$ and $\{x y z\}=\{x y z\}_{L t}$ is left $t$-standard.

7.4 REMARK. The same type of arguments based on Key Lemma 6.4 can be used to show for nonnuclear $t, s$ that

$$
\begin{aligned}
& \{x y 1\}=\left(x t^{-1}\right)(t y),\{x 1 z\}=x z,\{1 y z\}=\left(z t^{-1}\right)(t y) \quad \Rightarrow\{x y z\}=\left[(x z) t^{-1}\right](t y), \\
& \{x y 1\}=\left(y s^{-1}\right)(s x),\{x 1 z\}=x z,\{1 y z\}=\left(y s^{-1}\right)(s z) \Rightarrow\{x y z\}=\left(y s^{-1}\right)[s(x z)],
\end{aligned}
$$

etc.

Summarizing our results 5.8,6.1, 7.1 we have

7.5 TheOREM. Every unital composition triple is a permutation of a left or right $t$-standard triple $\{x y z\}=\left[\left(x t^{-1}\right)(t y)\right] z$ or $x\left[\left(y t^{-1}\right)(t z)\right]$ for an invertible element $t$ in a composition algebra.

If $t$ is nonnuclear we can pass to an isotope to obtain a standard triple as in (3.3). Using (4.2) and our classification of (3.4) of standard triples, we have our main

7.6 Classification Theorem. Every composition triple is isotopic to a standard composition triple on a unital composition algebra $C$, thus to one of the following types.

(I) $C=\Sigma$ inseparable field extension: $\{x y z\}=x y z$;

(II) $C=\Omega$ quadratic extension: $\{x y z\}=x y z$;

(III) $C=B$ quaternion: $\{x y z\}$ is one of
(i) $x y z$,
(ii) $x z y$,
(iii) $y x z$; 
(IV) C octonion: $\{x y z\}$ is one of

(i) $(x y) z$,

(ii) $(x z) y$,

(iii) $(y x) z$,

(iv) $x(y z)$,

(v) $x(z y)$,

(vi) $y(x z)$.

Two composition triples are isotopic iff they are associated with isomorphic composition algebras (i.e. equivalent norm forms) and have the same type $(N)$ and $(n)$ above.

If $\Phi$ is algebraically closed then there is precisely one composition algebra or quadratic form of each type I-IV, so there are precisely 11 nonisotopic composition triples. Similarly, if $\Phi=\mathbf{R}$ there are precisely 11 nonisotopic triples with positive definite forms $Q$ (the case of geometric interest).

\section{REFERENCES}

1. N. Jacobson, Composition algebras and their automorphisms, Rend. Circ. Mat. Palermo (2) 7 (1958), 55-80.

2. __ Triality and Lie algebras of type $D_{4}$, Rend. Circ. Mat. Palermo (2) 13 (1964), 1-25.

3. I. Kaplansky, Infinite-dimensional quadratic forms admitting composition, Proc. Amer. Math. Soc. 4 (1953), 956-960.

4. K. A. Zhevlakov, A. M. Slin'ko, I. P. Shestakov and A. I. Shirshov, Rings that are nearly associative, Nauka, Moscow, 1978. (Russian)

5. F. Van der Blij and T. A. Springer, Octaves and triality, Nieuw Arch. Wisk. (3) 8 (1960), 158-169.

Department of Mathematics, University of Virginia, Charlottesville, Virginia 22903 